\title{
Phase separation in a two-band model for strongly correlated electrons
}

\author{
A. O. Sboychakov, K. I. Kugel, and A. L. Rakhmanov \\ Institute for Theoretical and Applied Electrodynamics, \\ Russian Academy of Sciences, Izhorskaya Str. 13, Moscow, 125412 Russia
}

(Dated: November 12, 2018)

\begin{abstract}
The two-band Hubbard model is used to analyze a possibility of a non-uniform charge distribution in a strongly correlated electron system with two types of charge carriers. It is demonstrated that in the limit of strong on-site Coulomb repulsion, such a system has a tendency to phase separation into the regions with different charge densities even in the absence of magnetic or any other ordering. This tendency is especially pronounced if the ratio of the bandwidths is large enough. The characteristic size of inhomogeneities is estimated accounting for the surface energy and the electrostatic energy related to the charge imbalance.
\end{abstract}

PACS numbers: 75.47.Lx, 64.75.+g, 75.30.-m, 71.30.+h 


\section{INTRODUCTION}

The phase separation is commonly considered as an inherent property of strongly correlated electron systems ${ }^{1}$. Usually this phenomenon is treated as a result of a coexistence and competition of different kinds of ordering (magnetic, charge, orbital) $2, \underline{3}$. The most widely discussed type of the phase separation is a formation of nanoscale inhomogeneities such as ferromagnetic metallic droplets in an insulating antiferromagnetic material arising due to the self-trapping of charge carriers ${ }^{4}$. Such type of the phase separation is characteristic of the doped manganites. The another type of the nanoscale inhomogeneity is the modulation of the electron density due to antiferromagnetic correlations, which is considered as possible mechanism of the phase separation observed in superconducting cuprates ${ }^{5}, \underline{6}$.

Nevertheless, the phase separation can manifest itself even without some specific type of ordering, e.g., if the system contains different types of charge carriers. The simplest illustration of such a behavior gives the Falicov-Kimball model ${ }^{7}$, which is often used as a toy model for heavy-fermion compounds. This model describes the system of itinerant and localized electrons with a strong on-site Coulomb repulsion. The numerical simulations of this model demonstrated an inhomogeneous charge density distribution at some relation between the itinerant electron bandwidth and the distance between the localized level and the bottom of the $\operatorname{band}^{8}$. The competition between the metallicity and localization in a similar system with magnetic interactions was studied in Refs. 9, 10 with an emphasis on the phase diagram of magnetic oxides such as manganites. The system with a band and localized level is a limiting case of much more common situation of two bands with different width.

In this paper, we use a two-band Hubbard model for the description of a strongly correlated electron system with two types of charge carriers. We demonstrate that the phase separation in this system arises even without any ordering if the ratio of the bandwidths is large enough. In Section II, we write out the Hamiltonian of the model. In Section III, we study the electron structure of a homogeneous state. In Section IV, we analyze the possibility of the phase separation and estimate the size of inhomogeneity accounting for the long-range Coulomb interaction and surface energy. In Section V, we discuss the obtained results. 


\section{THE MODEL}

Let us consider a strongly correlated electron system with two bands $a$ and $b$ of different width. Let the first band, $a$, be wider than the second one, $b$. Such a system could be described by the following Hubbard Hamiltonian

$$
H=-\sum_{\langle\mathbf{i} \mathbf{j}\rangle \alpha, \sigma} t^{\alpha} a_{\mathbf{i} \alpha \sigma}^{\dagger} a_{\mathbf{j} \alpha \sigma}-\epsilon \sum_{\mathbf{i} \sigma} n_{\mathbf{i} b \sigma}-\mu \sum_{\mathbf{i} \alpha, \sigma} n_{\mathbf{i} \alpha \sigma}+\frac{1}{2} \sum_{\mathbf{i} \alpha, \sigma} U^{\alpha} n_{\mathbf{i} \alpha \sigma} n_{\mathbf{i} \alpha \bar{\sigma}}+\frac{U^{\prime}}{2} \sum_{\mathbf{i} \alpha, \sigma \sigma^{\prime}} n_{\mathbf{i} \alpha \sigma} n_{\mathbf{i} \bar{\alpha} \sigma^{\prime}} .
$$

Here, $a_{\mathbf{i} \alpha \sigma}^{\dagger}$ and $a_{\mathbf{i} \alpha \sigma}$ are the creation and annihilation operators for electrons in the bands $\alpha=\{a, b\}$ at site $\mathbf{i}$ with spin projection $\sigma$, and $n_{\mathbf{i} \alpha \sigma}=a_{\mathbf{i} \alpha \sigma}^{\dagger} a_{\mathbf{i} \alpha \sigma}$. The symbol $\langle\ldots\rangle$ denotes the summation over nearest-neighbor sites. The first term in Eq. (11) corresponds to the kinetic energy of the conduction electrons in bands $a$ and $b$ with the hopping integrals $t_{a}>t_{b}$. In our model, we ignore the interband hopping. The second term describes the shift $\epsilon$ of the center of band $b$ with respect to the center of band $a$. The last two terms correspond to the on-site Coulomb repulsion of two electrons either in the same state (with the Coulomb energy $U^{\alpha}$ ) or in the different states $\left(U^{\prime}\right)$. The bar above $\alpha$ or $\sigma$ denotes not $\alpha$ or not $\sigma$, respectively. The assumption of the strong electron correlations means that the Coulomb interaction is large, that is, $U^{\alpha}, U^{\prime} \gg t^{\alpha}, \epsilon$. The total number $n$ of electrons per site is a sum of electrons in the $a$ and $b$ states, $n=n_{a}+n_{b}$, and $\mu$ is the chemical potential. Below for definiteness sake, we consider the case $n \leq 1$.

\section{HOMOGENEOUS STATE}

The homogeneous state of the model formulated above can be analyzed by standard methods at arbitrary band filling $n$. Let us introduce a one-particle Green function

$$
G_{\alpha \sigma}\left(\mathbf{j}-\mathbf{j}_{0}, t-t_{0}\right)=-i\left\langle\hat{T} a_{\mathbf{j} \alpha \sigma}(t) a_{\mathbf{j}_{0} \alpha \sigma}^{\dagger}\left(t_{0}\right)\right\rangle
$$

where $\hat{T}$ is the time ordering operator. The equation of motion for the one-particle Green function with Hamiltonian (11) can be written as

$$
\begin{aligned}
& \left(i \frac{\partial}{\partial t}+\mu+\epsilon^{\alpha}\right) G_{\alpha \sigma}\left(\mathbf{j}-\mathbf{j}_{0}, t-t_{0}\right)=\delta_{\mathbf{j}_{0}} \delta\left(t-t_{0}\right)-t^{\alpha} \sum_{\Delta} G_{\alpha \sigma}\left(\mathbf{j}-\mathbf{j}_{0}+\Delta, t-t_{0}\right) \\
& +U^{\alpha} \mathcal{G}_{\alpha \sigma, \alpha \bar{\sigma}}\left(\mathbf{j}-\mathbf{j}_{0}, t-t_{0}\right)+U^{\prime} \sum_{\sigma^{\prime}} \mathcal{G}_{\alpha \sigma, \bar{\alpha} \sigma^{\prime}}\left(\mathbf{j}-\mathbf{j}_{0}, t-t_{0}\right),
\end{aligned}
$$


where $\epsilon^{\alpha}=0$ for $\alpha=a$ and $\epsilon^{\alpha}=\epsilon$ for $\alpha=b$, the summation in the second term in the righthand side of Eq. (3) is performed over sites nearest to $\mathbf{j}$, and $\boldsymbol{\Delta}$ are the vectors connecting the site $\mathbf{j}$ with its nearest neighbors. Equation (2) includes the two-particle Green functions of the form

$$
\mathcal{G}_{\alpha \sigma, \beta \sigma^{\prime}}\left(\mathbf{j}-\mathbf{j}_{0}, t-t_{0}\right)=-i\left\langle\hat{T} a_{\mathbf{j} \alpha \sigma}(t) n_{\mathbf{j} \beta \sigma^{\prime}}(t) a_{\mathbf{j}_{0} \alpha \sigma}^{\dagger}\left(t_{0}\right)\right\rangle
$$

Then, we should write the equations of motion for these functions, which will include the next order Green functions, etc. To cut such infinite chain of equations, we shall use here the following procedure.

In the limit of strong Coulomb repulsion, the presence of two electrons at the same site is unfavorable, and the two-particle Green function Eq. (4) is of the order of $1 / U$, where $U \sim U_{\alpha}, U^{\prime}$. The equation of motion for $\mathcal{G}_{\alpha \sigma, \beta \sigma^{\prime}}$ includes the three-particle terms coming from the commutator of $a_{\mathbf{j} \alpha \sigma}(t)$ with the $U$ terms of Hamiltonian (11) in the form $\left\langle\hat{T} a_{\mathbf{j} \alpha \sigma}(t) n_{\mathbf{j} \beta \sigma^{\prime}}(t) n_{\mathbf{j} \gamma \sigma^{\prime \prime}}(t) a_{\mathbf{j}_{0} \alpha \sigma}^{\dagger}\left(t_{0}\right)\right\rangle$. It is easy to see that these terms are of the order of $1 / U^{2}$ and in our approximation, we neglect them. In the equations of motion for the two-particle Green functions, we make the decoupling corresponding to the Hubbard I approximation ${ }^{11}$. That is, in term coming from the commutator of $a_{\mathbf{j} \alpha \sigma}(t)$ with the kinetic-energy terms of Hamiltonian (11), we make the following replacement $\left\langle\hat{T} a_{\mathbf{j}+\boldsymbol{\Delta} \alpha \sigma}(t) n_{\mathbf{j} \beta \sigma^{\prime}}(t) a_{\mathbf{j}_{0} \alpha \sigma}^{\dagger}\left(t_{0}\right)\right\rangle \rightarrow$ $\left\langle n_{\mathbf{j} \beta \sigma^{\prime}}\right\rangle\left\langle\hat{T} a_{\mathbf{j}+\boldsymbol{\Delta} \alpha \sigma}(t) a_{\mathbf{j}_{0} \alpha \sigma}^{\dagger}\left(t_{0}\right)\right\rangle$. The analogous decoupling in terms coming from the commutator of $n_{\mathbf{j} \alpha \sigma}(t)$ with the same kinetic-energy operator yields zero ${ }^{9}, 10,11$. As a result, the equations for the two-particle Green functions can be written as

$$
\begin{aligned}
\left(i \frac{\partial}{\partial t}+\mu+\epsilon^{\alpha}-U^{\alpha}\right) & \mathcal{G}_{\alpha \sigma, \alpha \bar{\sigma}}\left(\mathbf{j}-\mathbf{j}_{0}, t-t_{0}\right) \\
& =n_{\alpha \bar{\sigma}}\left[\delta_{\mathbf{j}_{0}} \delta\left(t-t_{0}\right)-t^{\alpha} \sum_{\boldsymbol{\Delta}} G_{\alpha \sigma}\left(\mathbf{j}-\mathbf{j}_{0}+\boldsymbol{\Delta}, t-t_{0}\right)\right] \\
\left(i \frac{\partial}{\partial t}+\mu+\epsilon^{\alpha}-U^{\prime}\right) & \mathcal{G}_{\alpha \sigma, \bar{\alpha} \sigma}\left(\mathbf{j}-\mathbf{j}_{0}, t-t_{0}\right) \\
= & n_{\bar{\alpha} \sigma}\left[\delta_{\mathbf{j}_{0}} \delta\left(t-t_{0}\right)-t^{\alpha} \sum_{\boldsymbol{\Delta}} G_{\alpha \sigma}\left(\mathbf{j}-\mathbf{j}_{0}+\Delta, t-t_{0}\right)\right]
\end{aligned}
$$

where $n_{\alpha \sigma}=\left\langle n_{\mathbf{j} \alpha \sigma}\right\rangle$ is the average number of electron per site in the state $(\alpha, \sigma)$.

Eqs. (3), (15), and (6) are the closed system for one- and two-particle Green functions. This system can be solved in a conventional manner $\underline{11}$ passing from the time-space to the frequency-momentum representation. Eliminating two-particle Green functions we can find 
the explicit expression for single-particle Green functions, which have poles corresponding to the Hubbard sub-bands for each band, $a$ or $b$. The splitting between these sub-bands is determined by on-site Coulomb repulsion $U^{\alpha}$ of electrons in the same states. The on-site Coulomb repulsion $U^{\prime}$ of electrons in different states gives rise to the correlation between the fillings of $a$ and $b$ bands (analogous to the case of localized and itinerant electrons discussed in Ref. 9, 10).

If the total number of the electrons per site does not exceed unity, $n \leq 1$, the upper Hubbard sub-bands are empty, and we can proceed to the limit $U_{\alpha}, U^{\prime} \rightarrow \infty$. In this case, the one-particle Green function $G_{\alpha \sigma}$ in the frequency-momentum representation can be written as

$$
G_{\alpha \sigma}(\mathbf{k}, \omega)=\frac{g_{\alpha \sigma}}{\omega+\mu+\epsilon^{\alpha}-g_{\alpha \sigma} w_{\alpha} \zeta(\mathbf{k})},
$$

where $w_{\alpha}=z t^{\alpha}, z$ is the number of nearest neighbors,

$$
g_{\alpha \sigma}=1-\sum_{\sigma^{\prime}} n_{\bar{\alpha} \sigma^{\prime}}-n_{\alpha \bar{\sigma}}
$$

and

$$
\zeta(\mathbf{k})=-\frac{1}{z} \sum_{\boldsymbol{\Delta}} \mathrm{e}^{i \mathbf{k} \boldsymbol{\Delta}}
$$

is the spectral function depending on the lattice symmetry. In the case of simple cubic lattice, we have

$$
\zeta(\mathbf{k})=-\frac{1}{3}\left[\cos \left(k^{1} d\right)+\cos \left(k^{2} d\right)+\cos \left(k^{3} d\right)\right],
$$

where $d$ is the lattice constant.

In the main approximation in $1 / U$ considered here, the magnetic ordering does not appear. To study the possible types of magnetic ordering in our model, it is necessary to take into account the higher order terms in $1 / U$. So, below we assume that

$$
n_{\alpha \uparrow}=n_{\alpha \downarrow} \equiv n_{\alpha} / 2
$$

From the expression for Green function (7), it follows that the filling of each lower sub-band is equal to $g_{\alpha \uparrow}+g_{\alpha \downarrow} \equiv 2 g_{\alpha}$. Using (8) and (10), we have

$$
g_{\alpha}=1-n_{\bar{\alpha}}-\frac{n_{\alpha}}{2} .
$$

Using the relation for the density of states $\rho_{\alpha}(E)=-\pi^{-1} \operatorname{Im} \int G_{\alpha}(\mathbf{k}, E+i 0) d^{3} \mathbf{k} /(2 \pi)^{3}$, we get the following expression for the number of electrons

$$
n_{\alpha}=2 g_{\alpha} n_{0}\left(\frac{\mu+\epsilon^{\alpha}}{g_{\alpha} w_{\alpha}}\right)
$$




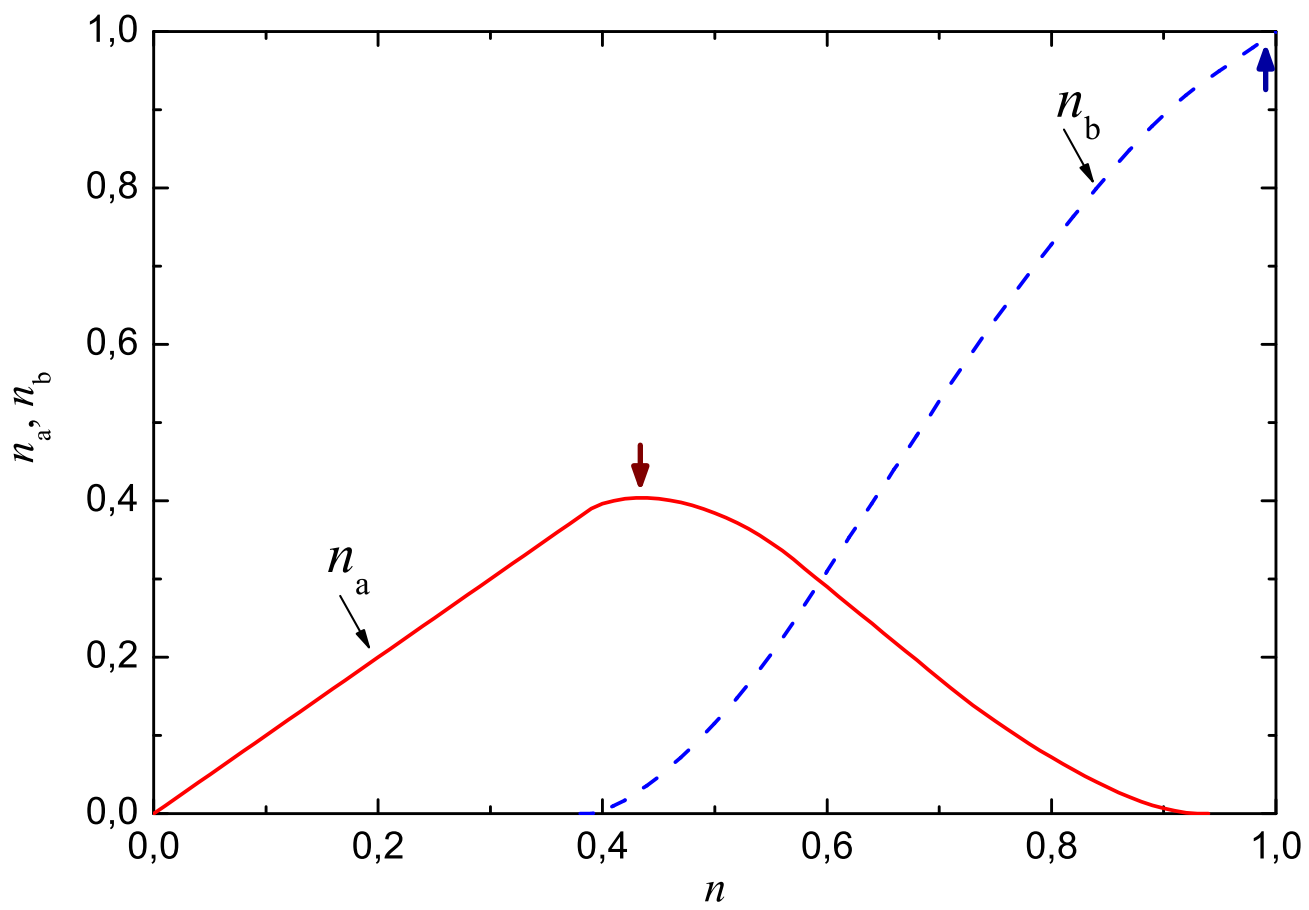

Figure 1: (Color online) $n_{a}$ (solid line) and $n_{b}$ (dashed line) vs the total number of charge carriers $n ; w_{b} / w_{a}=0.2, \epsilon / w_{a}=0.12$. Vertical arrows show the concentrations of $n_{a}$ and $n_{b}$ in the inhomogeneous state (see the text below).

where

$$
n_{0}\left(\mu^{\prime}\right)=\int_{-1}^{\mu^{\prime}} d E^{\prime} \rho_{0}\left(E^{\prime}\right)
$$

and

$$
\rho_{0}\left(E^{\prime}\right)=\int \frac{d^{3} \mathbf{k}}{(2 \pi)^{3}} \delta\left(E^{\prime}-\zeta(\mathbf{k})\right)
$$

is the density of states for free electrons (with the energy normalized by unity, $|E| \leq 1$ ). The chemical potential $\mu$ in Eq. (12) can be found from the equality $n=n_{a}+n_{b}$.

Let us consider the case when the energy difference $\epsilon$ between centers of $a$ and $b$ bands is not too large, that is, of the order of the width of $b$ band, $w_{b}$. In this case, there exist only $a$ electrons at low doping until the chemical potential reaches the bottom of the $b$ band $-\epsilon-w_{b}$ at some concentration $n_{c}$. At $n>n_{c}$, the $b$ electrons appear in the system, and the effective width of $a$ band, $W_{a}=2 w_{a} g_{a}\left(n_{a}, n_{b}\right)$, starts to decrease. The plots of $n_{a}, n_{b}$, and the effective bandwidth are shown in Fig. 1 and Fig. 2 as functions of $n$. In all calculations, we use the spectrum $\zeta(\mathbf{k})$ in the form (9). 


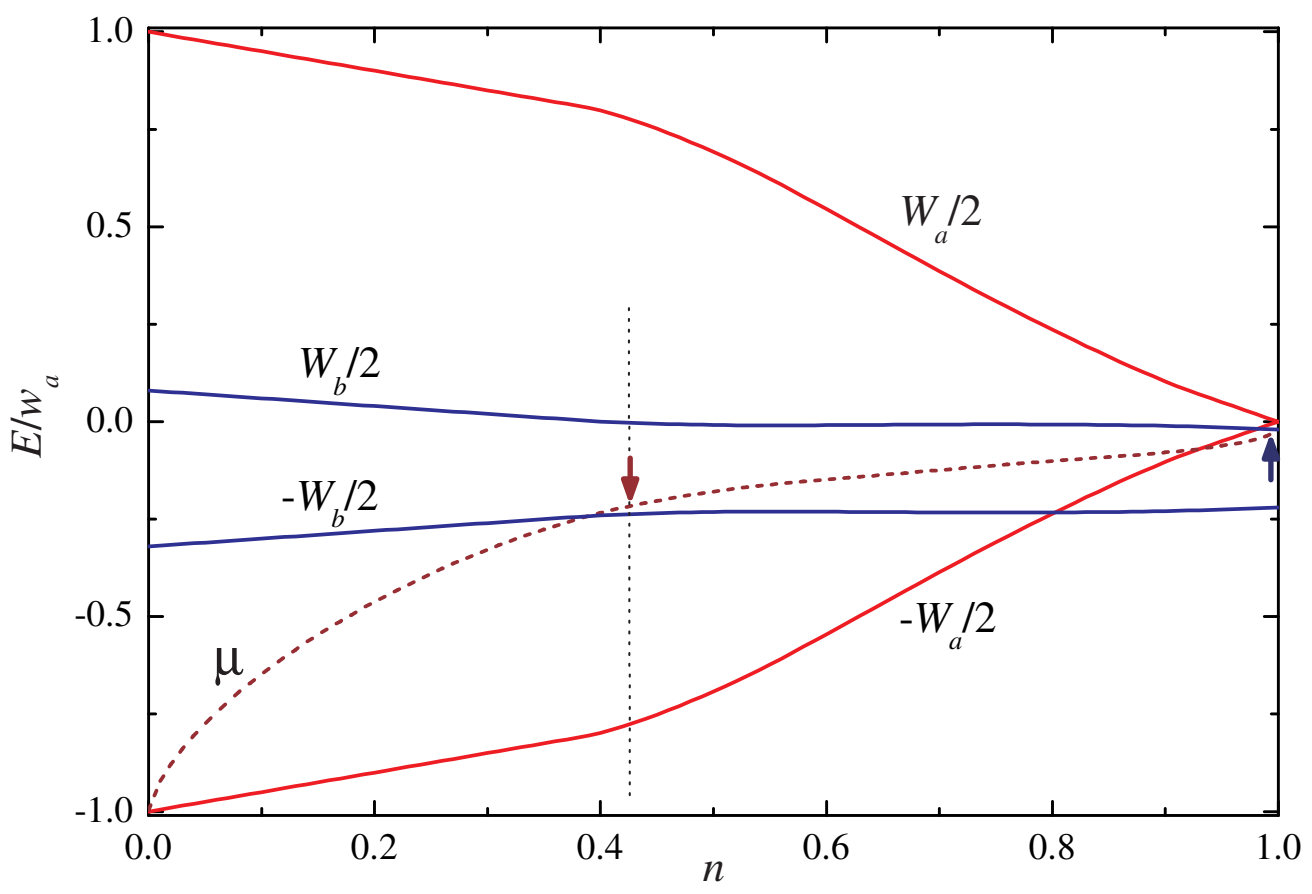

Figure 2: (Color online) Effective bandwidths $W_{\alpha}=2 w_{a} g_{\alpha}$ vs the total number of charge carriers $n$. The dashed curve is the chemical potential $\mu$. The values of the parameters are $w_{b} / w_{a}=0.2$, $\epsilon / w_{a}=0.12$. Vertical arrows show the concentrations of $n_{a}$ and $n_{b}$ in the inhomogeneous state.

The energy of the system in homogeneous state, $E_{\mathrm{hom}}$, is the sum of electron energies in all filled bands. Using the the density of states for free electrons Eq. (14) we can write $E_{\text {hom }}$ in the following form

$$
E_{\mathrm{hom}}=2 \sum_{\alpha} g_{\alpha}^{2} w_{\alpha} \varepsilon_{0}\left(\frac{\mu+\epsilon^{\alpha}}{g_{\alpha} w_{\alpha}}\right)-\epsilon n_{b},
$$

where

$$
\varepsilon_{0}\left(\mu^{\prime}\right)=\int_{-1}^{\mu^{\prime}} d E^{\prime} E^{\prime} \rho_{0}\left(E^{\prime}\right) .
$$

The dependence of $E_{\mathrm{hom}}(n)$ is shown in Fig. 3 by the solid line.

\section{PHASE SEPARATION}

\section{A. General consideration}

In this section, we analyze the possibility of the phase separation in the system. As one can see from Fig. 3, the energy for the homogeneous state, $E_{\text {hom }}(n)$, has two minima 
at different values of the charge carrier density. In this situation, it is favorable for a system to form two phases with different electron concentrations. Moreover, the existence of two minima is not a necessary condition for the formation of an inhomogeneous state and this phenomenon could be observed under more general conditions ${ }^{9}, 10$. However, the phase separation could be hindered by increase of the energy due to surface effects and a charge redistribution.

At first, we do not take into account the charge redistribution and surface terms in the total energy. In this way, we determine the optimum content of each phase and the corresponding charge densities but we can not find the characteristic size of the inhomogeneities.

We consider two phases, I (low carrier density) and II (high carrier density), with the number of electrons per site $n_{1}$ and $n_{2}$, respectively. A fraction $p$ of the system volume is occupied by the phase I and $1-p$ is a fraction of the phase II. We seek a minimum of the system energy

$$
E_{\mathrm{ps}}^{0}\left(n_{1}, n_{2}\right)=p E_{\mathrm{hom}}\left(n_{1}\right)+(1-p) E_{\mathrm{hom}}\left(n_{2}\right)
$$

under the condition of the charge carrier conservation

$$
n=p n_{1}+(1-p) n_{2}
$$

The results of calculations of the system energy in the phase separated state are shown in Fig. 3 for $w_{b} / w_{a}=0.2$ and $\varepsilon / w_{a}=0.12$ by the dashed curve. From this figure we see, that the phase separation exists in the range of $n$ where both types of charge carriers coexist in the homogeneous state. The numerical analysis shows that the concentrations of the charge carriers in each phase, $n_{1}$ and $n_{2}$, vary slowly with $n$, remaining close to certain optimal values for each phase: $n_{1} \approx n_{a} \approx 0.5$ for the state with low carrier density, whereas $n_{2} \approx n_{b} \approx 1$ for the state with high carrier density. The phase II can be considered as a MottHubbard insulator since the corresponding lower Hubbard sub-band is almost completely filled. If $n$ increases from zero to one, the phase separation may be favorable when the $n$ achieves the value corresponding to the energy minimum for the homogeneous state. At this value of $n$ the content of the phase I $p(n)$ starts to decrease from $p(n)=1$. With the further increase of $n, p(n)$ tends to zero at $n \approx n_{2}$. Therefore, we can conclude that the system may separate into metallic and insulating phases in a certain parameter range. An indication to the phase separation is a negative curvature of the $E_{\text {hom }}(n)$ curve at the right side from the energy minimum, see Fig. 3. 
The above discussion demonstrates that the width and filling of one band depends on the width and filling of other bands. The phase separation gives the possibility to attain the minimum free energy by an optimum filling of the electron bands. The phase separation can be favorable only if the bands are appreciably different. In Fig. 4 we plot the energy of the homogeneous state versus $n$ at different values of ratio $w_{b} / w_{a}$. We see that $\partial^{2} E / \partial n^{2}<0$ in a wide range of $n$ for $w_{b} / w_{a} \ll 1$, which indicates the possibility of the phase separation (see also Fig. 3). The phase separation becomes unfavorable only if $w_{b} / w_{a} \lesssim 0.4$.

\section{B. Characteristic size of inhomogeneities}

The phase separation leads to redistribution of charge carriers $\left(n_{1} \neq n_{2}\right)$. Therefore, we should take into account the electrostatic contribution to the total energy of the phaseseparated state. This contribution depends on the shape of inhomogeneities. Following Refs. 9,10,12, we consider a spherical geometry of the inhomogeneous state, namely, at $p<0.5$, the sample is modelled as an aggregate of spheres of phase I embedded into the matrix of phase II and vice versa for $p>0.5$. The electrostatic, $E_{C}$, energy is calculated in the Wigner-Seitz approximation, that is, we consider a set of spherical unit cells with zero total charge, where the spherical core of one phase is surrounded by a shell of another phase. Following the approach described in Refs. 10,12 we find at $p<0.5$

$$
E_{\mathrm{C}}=\frac{2 \pi e^{2}}{5 \epsilon d}\left(n_{1}-n_{2}\right)^{2}\left(\frac{R_{s}}{d}\right)^{2} p\left(2-3 p^{1 / 3}+p\right)
$$

where $\epsilon$ is the average permittivity and $R_{s}$ is the radius of the droplet of the phase I. In the case $p>0.5$, we should replace $n_{1} \leftrightarrow n_{2}$ and $p \leftrightarrow 1-p$.

Another contribution to the total energy depending of the size of inhomogeneities is related to the surface between two phases. It comes from the size quantization and depends on the electron densities in both phases. The case when one of the densities is zero was considered in Ref. 10. The generalization of this approach for non-zero densities is presented in Appendix, where surface energy $\sigma\left(n_{1}, n_{2}\right)$ is calculated using the perturbative approach proposed in Ref. 13. The corresponding contribution, $E_{S}$, to the total energy is proportional to the surface area between phases I and II. At $p<0.5$, it can be written in the form

$$
E_{\mathrm{S}}=p \frac{3 d}{R_{s}} \sigma\left(n_{1}, n_{2}\right)
$$




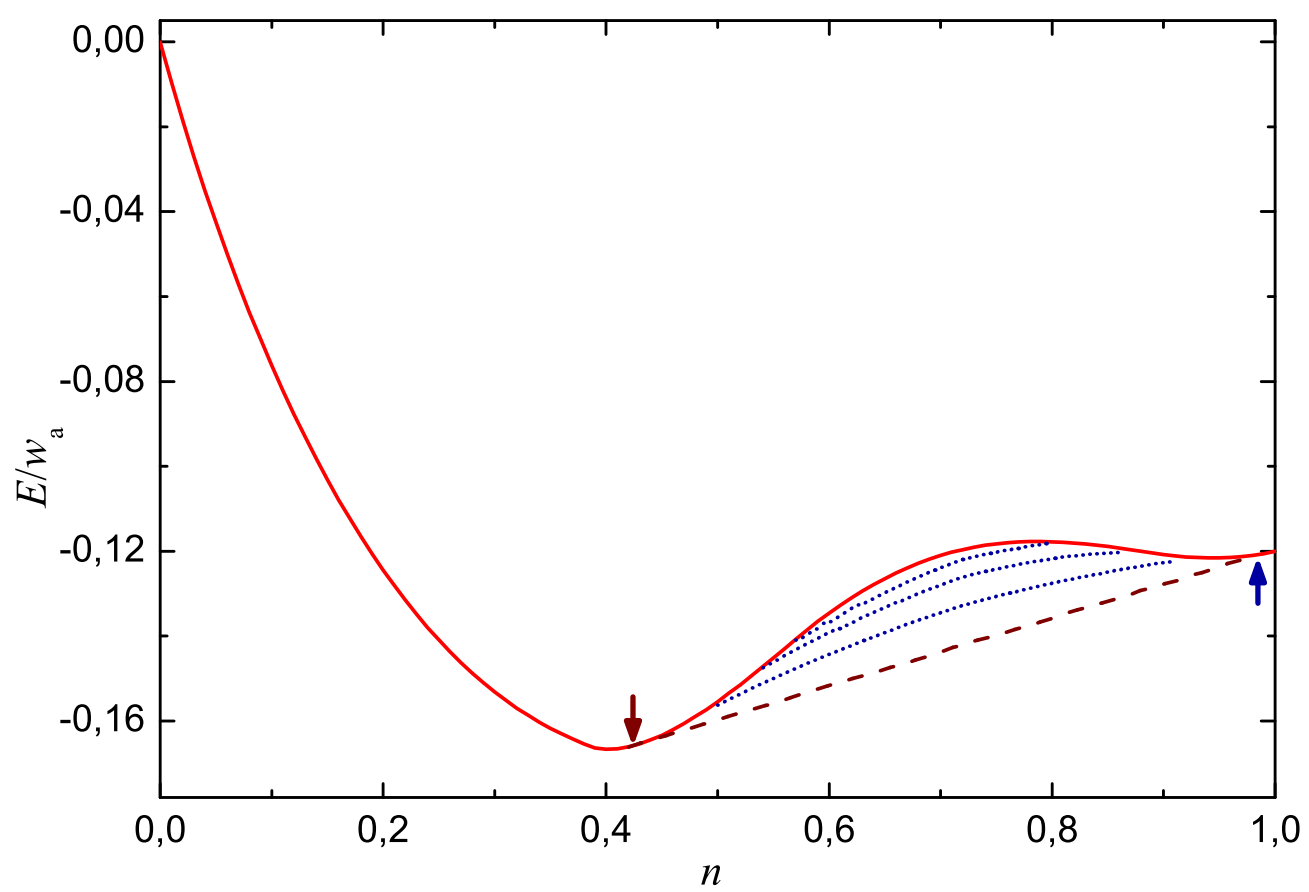

Figure 3: (Color online) The energy of the system $v s$ doping level $n$. Solid curve corresponds to the homogeneous state, whereas dashed curve is the energy of phase-separated state without taking into account electrostatic and surface contributions to the total energy. Dot curves are the energies of inhomogeneous state, Eqs. (22) and (23) , at $V_{0} / w_{a}=0.1,0.05,0.01$ from top to bottom (see text below). Here, $w_{b} / w_{a}=0.2$ and $\varepsilon / w_{a}=0.12$. Vertical arrows show the concentrations of $n_{a}$ and $n_{b}$ in the inhomogeneous state.

In the case $p>0.5$, we should replace $p \rightarrow 1-p$.

Minimization of the sum $E_{\mathrm{CS}}=E_{\mathrm{C}}+E_{\mathrm{S}}$ with respect to $R_{s}$ allows us to calculate this value. In doing so, we get at $p<0.5$

$$
R_{s}=d\left(\frac{15 \sigma\left(n_{1}, n_{2}\right)}{4 \pi V_{0}\left(n_{2}-n_{1}\right)^{2}\left(2-3 p^{1 / 3}+p\right)}\right)^{1 / 3}
$$

The total energy of the inhomogeneous state then reads

$$
E_{\mathrm{ps}}\left(n_{1}, n_{2}\right)=p E_{\mathrm{hom}}\left(n_{1}\right)+(1-p) E_{\mathrm{hom}}\left(n_{2}\right)+E_{\mathrm{CS}}\left(n_{1}, n_{2}\right)
$$

where

$$
E_{\mathrm{CS}}=3\left(V_{0} \frac{9 \pi}{10}\left(n_{2}-n_{1}\right)^{2} \sigma^{2}\left(n_{1}, n_{2}\right)\right)^{1 / 3} p\left(2-3 p^{1 / 3}+p\right)^{1 / 3}
$$

$V_{0}=e^{2} / \epsilon d$. The energy $E_{\mathrm{ps}}$ calculated by minimization of Eq. (22) with respect to $n_{1}$ and $n_{2}$ at different values of $V_{0}$ are shown in Fig. 3 by dot-dashed curves. We see, that the 


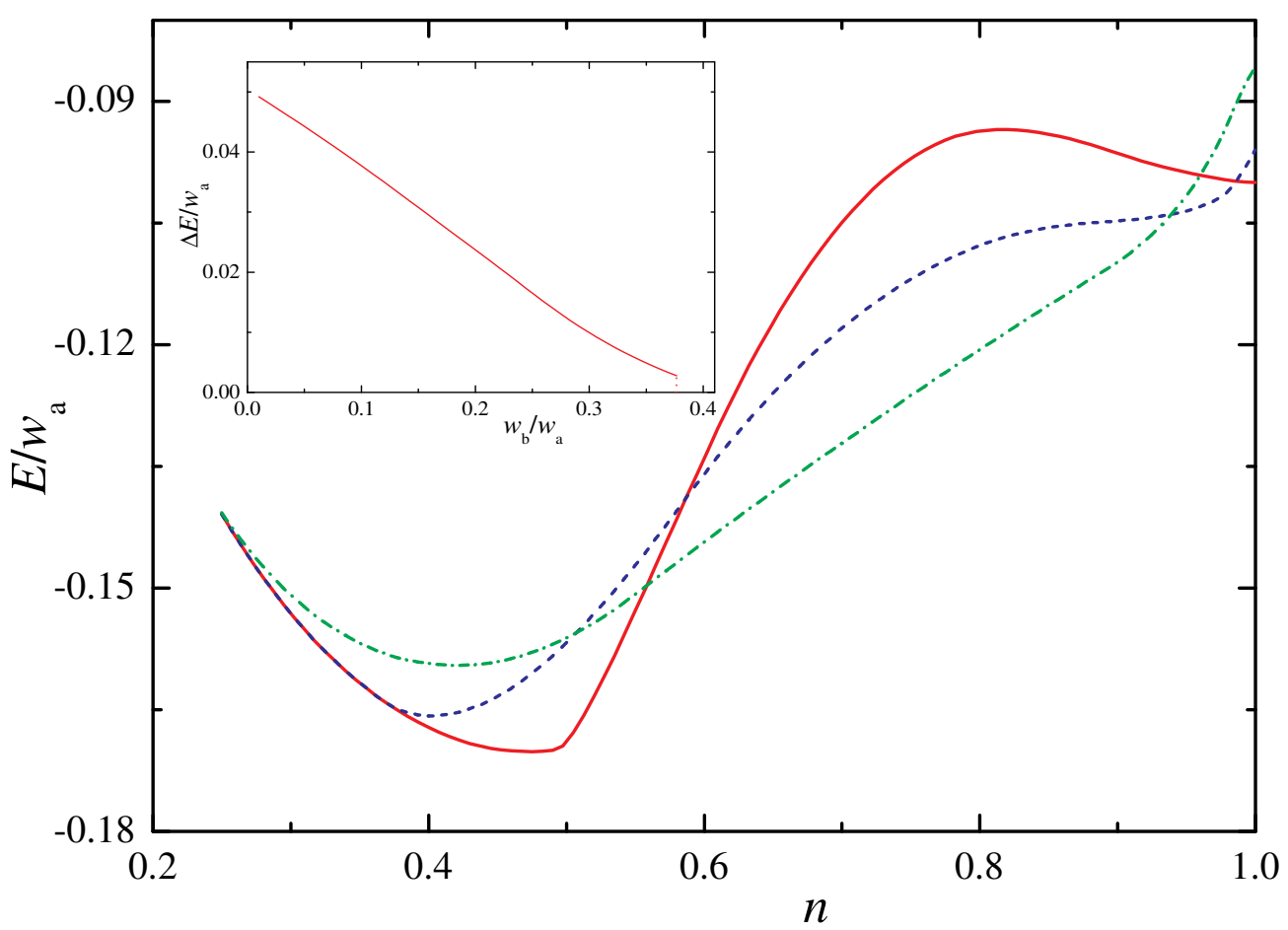

Figure 4: (Color online) The energy of homogeneous state $v s$ doping level $n$ at $w_{b} / w_{a}=0.1$ (solid line), $w_{b} / w_{a}=0.25$ (dashed line), and $w_{b} / w_{a}=0.4$ (dot-dashed line). The parameter $\varepsilon_{b} / w_{a}=0.1$ for all cases. The phase separation is favorable for solid and dashed curves in the range of doping $0.45 \lesssim n \lesssim 1$, where $\partial^{2} E / \partial n^{2}<0$, whereas for dot-dashed curves, only the homogeneous state exists. In the inset, the maximum energy gain due to the formation of the inhomogeneous state $\left(V_{0}=0, \varepsilon_{b} / w_{a}=0.1\right)$ as a function of the ratio $w_{b} / w_{a}$ is shown. For $w_{b} / w_{a} \gtrsim 0.38$ the phase separation becomes unfavorable in energy.

Coulomb repulsion in the system with an inhomogeneous charge distribution reduces the range of $n$, in which the phase separation is favorable. In Fig. 5 we plot the characteristic radius of inhomogeneities, $R_{s}$, as a function of $n$.

\section{DISCUSSION}

Thus, the phase separation can be favorable for the system of the strongly correlated electrons even in the absence of any specific ordering. We demonstrated that the state with inhomogeneous charge distribution can arise if there exist two types of the charge carriers with different bandwidth. The electron correlations due to on-site Coulomb repulsion lead 


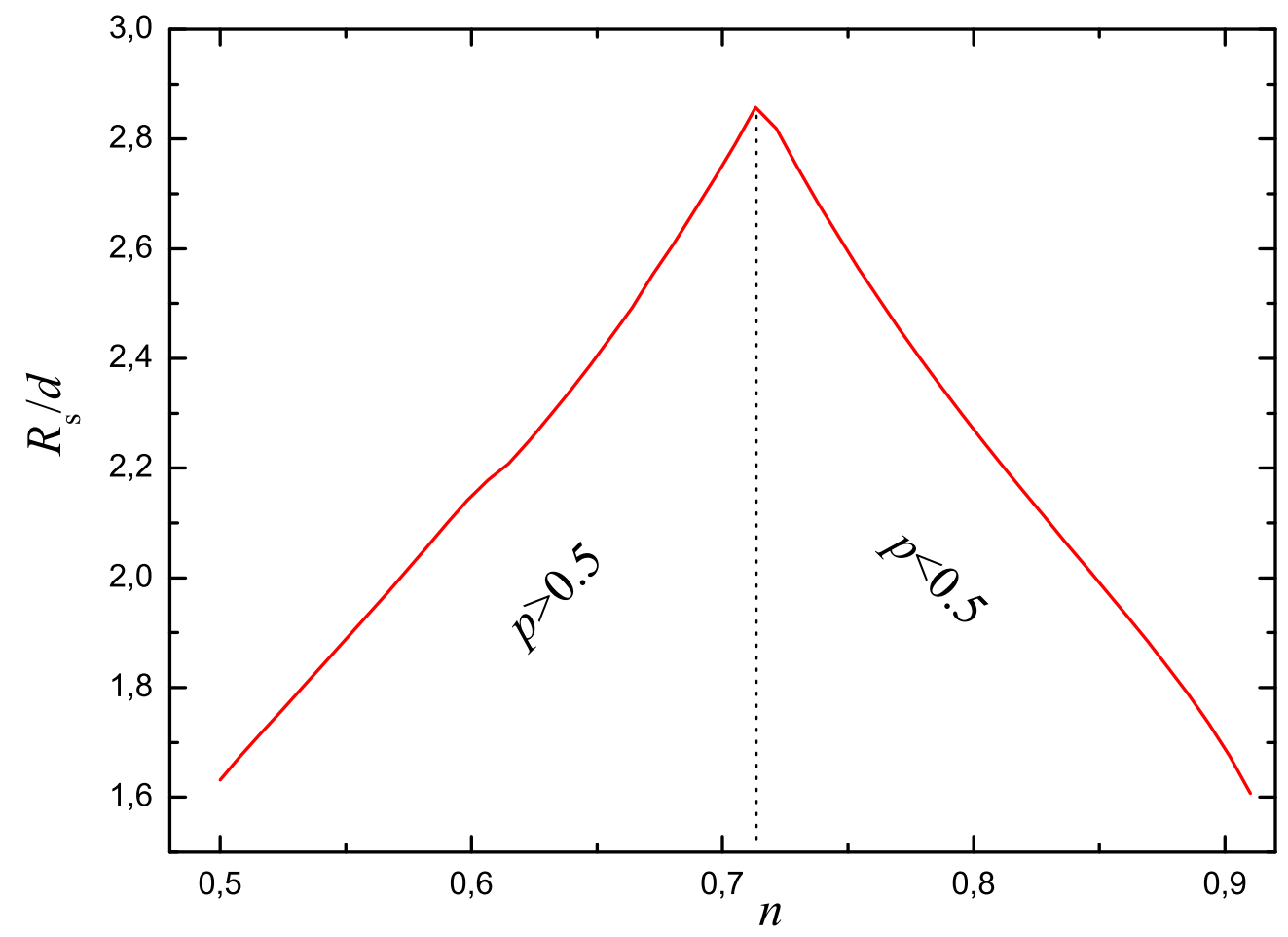

Figure 5: (Color online) The radius of droplets $R_{s}$ vs doping level $n$ at $w_{b} / w_{a}=0.2, \epsilon / w_{a}=0.12$, and $V_{0} / w_{a}=0.02$.

to the dependence of the bandwidth for one type of electrons on the band filling for another type of electrons. As a result, the dependence of energy on the total number of the charge carriers becomes non-monotonic. The competition between kinetic and correlation energies triggers the formation of an inhomogeneous ground state. It is particularly evident if the energy of the system as a function of electron density has two minima. In this case, it could be favorable for the system to separate into two states with electron densities close to these minima rather than to form a homogeneous state with an intermediate density. Such a situation is illustrated in Fig. 3.

It is clear that the phase separation can occur only if the bandwidths corresponding to two types of the charge carriers are sufficiently different, that is, the ratio $w_{b} / w_{a}$ of the widths of narrow and wide band should be rather small. The second condition is that the narrow and wide bands should not be widely separated from each other, that is, the ratio $\epsilon / w_{a}$ of the distance between the band centers and the width of the wider band should be less than unity. Naturally, the long-range electrostatic forces prevent nonuniform charge 
distribution and the condition $V_{0} / w_{a} \ll 1$ should be met. As it can be seen from Fig. 4, the phase separation can be favorable even if $w_{b} / w_{a} \lesssim 0.3-0.4$.

\section{Acknowledgments}

The work was supported by the European project CoMePhS, International Science and Technology Center, grant no. G1335, and Russian Foundation for Basic Research, project no. 05-02-17600.

\section{Appendix: SURFACE ENERGY}

The electrons in the phase-separated state are confined within a restricted volume $V_{s}$. This gives rise to the change in the density of states in both phases. At small ratio $\Delta=$

$S_{s} d / V_{s}$, where $S_{s}$ is the surface area of the sample, the density of states for free electrons can be written as $\underline{10}, 13$

$$
\rho\left(E^{\prime}\right)=\left(1+\frac{\Delta}{2}\right) \rho_{0}\left(E^{\prime}\right)-\frac{\Delta}{4}\left(\rho_{0}^{(2 D)}\left(E^{\prime}+1 / 3\right)+\rho_{0}^{(2 D)}\left(E^{\prime}-1 / 3\right)\right) .
$$

where $\rho_{0}$ is given by Eq. (14). Here $\rho_{0}^{(2 D)}$ is the density of states in two dimensions. Using this expression instead of (14), and expanding the Eqs. (12), (13), (15), and (16) in a series in powers of $\Delta$ up to the first order, we derive formula (20) with the correction for the size quantization $\sigma(n)$ in the form

$$
\begin{aligned}
\sigma=2 & \sum_{\alpha} g_{\alpha}^{(0)} w_{\alpha}\left[\varepsilon_{0}\left(\mu_{\alpha}^{\prime(0)}\right) n_{\alpha}^{(1)}+g_{\alpha}^{(0)} \delta \varepsilon_{0}\left(\mu_{\alpha}^{\prime(0)}\right)\right]+ \\
& \sum_{\alpha} g_{\alpha}^{(0)} w_{\alpha} \mu_{\alpha}^{\prime(0)} \rho_{0}\left(\mu_{\alpha}^{\prime(0)}\right)\left(\frac{2 \mu^{(1)}}{w_{\alpha}}-\mu_{\alpha}^{(0)} n_{\alpha}^{(1)}\right)-\sum_{\alpha} \epsilon n_{b}^{(1)}
\end{aligned}
$$

where

$$
\begin{gathered}
\mu_{\alpha}^{\prime(0)}=\frac{\mu^{(0)}+\epsilon^{\alpha}}{g_{\alpha}^{(0)} w_{\alpha}}, \\
n_{\alpha}^{(1)}=\frac{1}{2} \frac{\frac{4}{w_{\alpha}} \rho_{0}\left(\mu_{\alpha}^{\prime(0)}\right) \mu^{(1)}+g_{\alpha}^{(0)}\left[2 n_{0}\left(\mu_{\alpha}^{\prime(0)}\right)-n_{0}^{(2 d)}\left(\mu_{\alpha}^{\prime(0)}+\frac{1}{3}\right)-n_{0}^{(2 d)}\left(\mu_{\alpha}^{\prime(0)}-\frac{1}{3}\right)\right]}{1+\mu_{\alpha}^{(0)} \rho_{0}\left(\mu_{\alpha}^{\prime(0)}\right)-n_{0}\left(\mu_{\alpha}^{\prime(0)}\right)}, \\
\delta \varepsilon_{0}\left(\mu^{\prime}\right)=\frac{1}{2} \varepsilon_{0}\left(\mu^{\prime}\right)-\frac{1}{4}\left[\varepsilon_{0}^{(2 D)}\left(\mu^{\prime}+\frac{1}{3}\right)+\varepsilon_{0}^{(2 D)}\left(\mu^{\prime}-\frac{1}{3}\right)\right]+\frac{1}{12}\left[n_{0}^{(2 D)}\left(\mu^{\prime}+\frac{1}{3}\right)-n_{0}^{(2 D)}\left(\mu^{\prime}-\frac{1}{3}\right)\right],
\end{gathered}
$$


correction to the chemical potential $\mu^{(1)}$ is found from the condition $\sum_{\alpha} n_{\alpha}^{(1)}=0$, and the superscript (0) denotes the unperturbed value of corresponding quantity. The functions $n_{0}^{(2 D)}\left(\mu^{\prime}\right)$ and $\varepsilon_{0}^{(2 D)}\left(\mu^{\prime}\right)$ in these expressions are determined by Eqs. (13) and (16), respectively, where one should change $\rho_{0}$ to $\rho_{0}^{(2 D)}$. The surface energy $\sigma\left(n_{1}, n_{2}\right)$ per unit area between phases I and II is the sum $\sigma\left(n_{1}\right)+\sigma\left(n_{2}\right)$.

1 E. Dagotto, Science 309, 257 (2005).

2 E. Dagotto, Nanoscale Phase Separation and Colossal Magnetoresistance: The Physics of Manganites and Related Compounds (Springer-Verlag, Berlin, 2003).

3 M. Yu. Kagan and K. I. Kugel, Usp. Fiz. Nauk. 171, 577 (2001) [Physics - Uspekhi 44, 553 (2001)].

4 E. L. Nagaev, Pis'ma v ZhETF 6, 484 (1967) [JETP Lett. 6, 18 (1967)].

5 E.L. Nagaev, Usp. Fiz. Nauk 165, 529 (1995) [Physics - Uspekhi 38, 497 (1995)].

6 S. A. Kivelson and V. J. Emery, in Strongly Correlated Electronic Materials: The Los Alamos Symposium 1993, edited by K. S. Bedell et al.

7 L. M. Falicov and J. C. Kimball, Phys. Rev. Lett. 22, 997 (1969).

8 J. K. Freericks, Ch. Gruber, and N. Macris, Phys. Rev. B 60, 1617 (1999); J. K. Freericks, E. H. Lieb, and D. Ueltschi, Phys. Rev. Lett. 88, 106401 (2002); M. M. Maśka and K. Czajka, phys. stat. sol. (b) 242, 479 (2005).

9 K.I. Kugel, A.L. Rakhmanov, and A.O. Sboychakov, Phys. Rev. Lett. 95, 267210 (2005).

10 A.O Sboychakov, K.I. Kugel, and A.L. Rakhmanov, Phys. Rev. B 74, 026625 (2006).

11 J. Hubbard, Proc. Roy. Soc. (London) A276, 238 (1963); A281, 401 (1964).

12 J. Lorenzana, C. Castellani, and C. di Castro, Europh. Lett. 57, 704 (2002).

13 R. Balian and C. Bloch, Ann. Phys. (N.Y.) 60, 401 (1970). 